\title{
Elektrochemotherapie „durchlöchert“ Hauttumoren
}

— „An erster Stelle der Therapieoptionen bei Hautmetastasen steht die Operation“, erklärte Prof. Uwe Reinhold, Bonn. Allerdings ist diese nicht immer möglich, etwa bei ausgedehntem kutanen metastasierten Karzinom. Auch auf systemische und intraläsionale Chemo- bzw. Immuntherapie sprechen längst nicht alle intradermalen Metastasen an. Als neue Methode für die Therapie solider Primärtumoren der Haut wie auch zur lokalen Tumorkontrolle von Metastasen stellte der Dermatoonkologe die Elektrochemotherapie (ECT) vor. Dabei induziert man mittels eines kurzen elektrischen Impulses die Öffnung der Hautporen, was die kurzfristige Aufnahme eines Medikaments, etwa eines Zytostatikums, erleichtert. „Andere europäische Länder wie
Italien oder Spanien setzen dieses Verfahren schon viel länger ein. Wir sind jetzt dabei, es auch in Deutschland einzuführen“, berichtete Reinhold. Die Ansprechraten seien mit einer Komplettremissionsrate von $70-80 \%$ pro Sitzung relativ hoch. „Man kann die Methode öfter wiederholen und auch bei größeren Flächen einsetzen, unter dem Ziel der lokalen und häufig palliativen Tumorkontrolle“, erklärte Reinhold. Vorteilhaft ist, dass die ECT bei jeder Form von Hautkrebs oder Hautmetastasen anwendbar ist, man sie ambulant durchführen kann und keine signifikanten Nebenwirkungen auftreten.

mha

Vortrag: Hautmetastasen bei metastasierenden soliden Tumoren

\section{Rasante Zunahme des Merkelzell-Karzinoms}

- Das Merkelzell-Karzinom (MCC) ist nicht leicht zu erkennen, da es kaum besondere Wachstumsmerkmale aufweist. Doch die Inzidenz dieses Tumors steigt derzeit rapide an. Charakteristisch ist, dass es in der Regel auf chronisch UV-exponierter Haut, sehr schnell wächst und meist bei älteren sowie immunsupprimierten Menschen vorkommt. Dass das Immunsystem eine wichtige Rolle spielt, spiegelt sich auch in der Tatsache wider, dass eine histologisch gesicherte, komplette spontane Regression möglich ist. „Wir hatten vor kurzem einen Patienten, dessen Tumor von fast drei Zentimetern Durchmesser bei der Wiedervor-

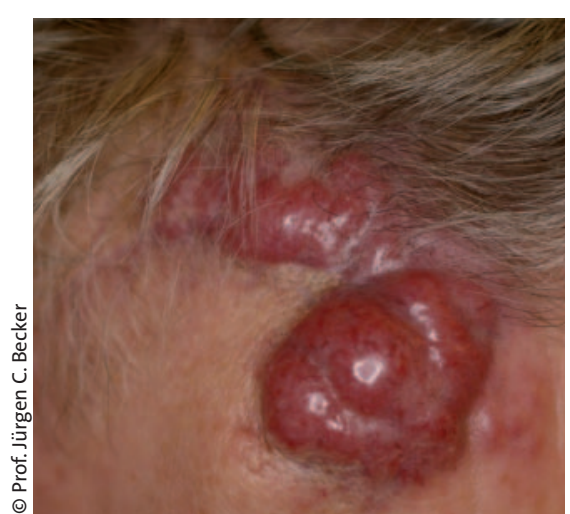

Abb. 1: Lokalrezidiv eines MCC an der Stirn. stellung komplett verschwunden war", berichtete Prof. Jürgen C. Becker, Würzburg. Dementsprechend weisen Patienten mit entzündlichem Infiltrat in der Neoplasie eine bessere Prognose auf als Betroffene mit geringer Immunantwort im Tumor. Zur Ausbreitungsdiagnostik gehören die Sonografie der drainierenden Lymphknotenstationen, des Abdomens sowie ein Röntgen-Thorax. Zunehmend setzt sich auch die Biopsie des Sentinel-Lymphknotens durch, die in etwa $30 \%$ positive Ergebnisse liefert. Als weitere Möglichkeit nannte Becker das [68Ga]-DOTATOC-PET, mit welchem sich Somatostatinrezeptoren auf der Oberfläche der Tumorzellen nachweisen lassen.

Als Primärtherapie gilt die Exzision mit einem Sicherheitsabstand von $2-3 \mathrm{~cm}$. Eine adjuvante Radiatio der Tumorregion und des regionären Lymphabflussgebietes wird empfohlen, da sehr häufig Intransit- und Lymphknoten-Metastasen vorkommen. Bei positiven Sentinel-Lymphknoten oder klinischem Befall (Makrometastasen) ist eine Lymphknoten-Dissektion durchzuführen. Laut Leitlinien erfordern Fernmetastasen eine systemische Therapie z.B. mit Anthrazyklinen.

mha

Vortrag: Neues zum Merkelzell-Karzinom 